\title{
New type phase transitions in the Mitsui model with bimodal random field
}

\author{
I.V.Stasyuk, O.V.Velychko \\ Institute for Condensed Matter Physics \\ of the National Academy of Sciences of Ukraine, \\ 1 Svientsitskii Str., 79011 Lviv, Ukraine
}

Received December 25, 1998

Thermodynamic functions of the Mitsui model with bimodal random field are calculated in the mean field approximation for long-range interactions with an exact treatment of the intracell interaction of pseudospins. Conditions of the appearing of the first order phase transition between nonequivalent nonpolar phases or the possibility of phase separation are investigated.

Key words: Mitsui model, random field, vacancies, phase transitions, HTSC

PACS: $64.60 . \mathrm{Cn}, 74.62 . \mathrm{Dh}, 74.72 . \mathrm{Bk}$

\section{Introduction}

A crystalline system formed by two sublattices of pseudospins in equal value longitudinal fields with opposite signs (Mitsui model) affected by vacancies randomly situated in the third sublattice placed in the symmetrical position is considered. The vacancy influence is modelled by the random fields with the bimodal distribution acting onto pseudospins. Both equilibrium and nonequilibrium distributions of vacancies are considered.

This model is of pragmatic interest due to the possibility of describing the apex oxygen - chain oxygen subsystem in nonstoichiometric $\mathrm{YBa}_{2} \mathrm{Cu}_{3} \mathrm{O}_{7-x}$ high- $\mathrm{T}_{\mathrm{c}}$ superconductor. In the framework of the model, anharmonic vibrations of apex oxygen ions in double wells [1-3] are described by pseudospins [4] and chain oxygen vacancies give rise to the random field.

Main goals of the present paper are: to consider the effect of an internal longitudinal random field on thermodynamics of Mitsui model; to propose a reliable approach for calculating average pseudospin values which takes into account a presumably strong interaction of pseudospins from different sublattices in the same cell; to investigate a phase diagram of the model and to establish the regions of model parameter values where the system has got phase transitions or becomes unstable (manifests a bistable behaviour [5] or a phase separation). 


\section{Nonequilibrium vacancy distribution}

The Hamiltonian of the two-sublattice pseudospin model under consideration is as follows

$$
H=-\sum_{i} h_{i}\left(S_{1 i}^{z}-S_{2 i}^{z}\right)-\frac{1}{2} \sum_{i} \sum_{j} j_{11}(i, j)\left(S_{1 i}^{z} S_{1 j}^{z}+S_{2 i}^{z} S_{2 j}^{z}\right)-\sum_{i} \sum_{j} j_{12}(i, j) S_{1 i}^{z} S_{2 j}^{z} .
$$

Here $S_{\alpha i}^{z}= \pm \frac{1}{2}$ are the values of pseudospin $z$-component (which corresponds, e.g. to two possible positions of anharmonic $\mathrm{O}(4)$ ion) from $\alpha$ sublattice $(\alpha=1,2)$ in the $i$-th unit cell. The first term has a meaning of interaction with some internal field acting on pseudospins of different sublattices in the opposite directions. The two next terms describe interactions between pseudospins located in the same and in the different sublattices.

The random field probability distribution looks like

$$
P\left(h_{i}\right)=c \delta\left(h_{i}-h\right)+(1-c) \delta\left(h_{i}-h_{\mathrm{vac}}\right)=\sum_{n=1}^{2} p_{n} \delta\left(h_{i}-h_{n}\right)
$$

where $p_{1}=c$ and $p_{2}=1-c$. The correlations between random fields on different cells are not included. For this reason such a distribution function can be applied in the case of $\mathrm{YBa}_{2} \mathrm{Cu}_{3} \mathrm{O}_{7-x}$ at sufficiently small values of $x$ (ortho-I phase, $x<0.15$ ), when the ordering processes of vacancies have not yet taken place and its distribution is random.

Phase transitions in the Mitsui model may be analyzed in the mean field approximation (MFA) and in the cluster approximation [6-8]. If an interaction between the nearest neighbours from different sublattices is presumed to be significant it should be treated separately and a cell formed by the nearest pseudospins from different sublattices with the same site index should be considered as the basic one. To do this the basis of four states of the pair of pseudospins in the cell $\left|S_{1 i}^{z} S_{2 i}^{z}\right\rangle$ is introduced $[9]$

$$
|1\rangle=|++\rangle, \quad|2\rangle=|+-\rangle, \quad|3\rangle=|-+\rangle, \quad|4\rangle=|--\rangle .
$$

As the next step, pseudospin variables are expressed in terms of Hubbard operators acting in the space of these states. Now one can treat intracell interactions exactly while intercell interactions can be treated in the MFA.

The full (thermodynamical and configurational) averaging is made in the MFA manner. In particular this means that the acting on the site mean field does not depend on the configuration of environment [10]. As a result, a set of simultaneous equations for variables $\eta$ and $\xi$ is obtained

$$
\left\{\begin{array}{l}
\eta=\frac{1}{2} \sum_{n=1}^{2} p_{n}\left[\exp \left(\beta \lambda_{n 1}\right)-\exp \left(\beta \lambda_{n 4}\right)\right]\left[\sum_{k=1}^{4} \exp \left(\beta \lambda_{n k}\right)\right]^{-1} \\
\xi=\frac{1}{2} \sum_{n=1}^{2} p_{n}\left[\exp \left(\beta \lambda_{n 2}\right)-\exp \left(\beta \lambda_{n 3}\right)\right]\left[\sum_{k=1}^{4} \exp \left(\beta \lambda_{n k}\right)\right]^{-1}
\end{array}\right.
$$


where

$$
\begin{aligned}
& \eta=\frac{1}{2}\left(\left\langle S_{1}^{z}\right\rangle+\left\langle S_{2}^{z}\right\rangle\right), \xi=\frac{1}{2}\left(\left\langle S_{1}^{z}\right\rangle-\left\langle S_{2}^{z}\right\rangle\right), a=\frac{j_{12}(0)-j_{11}(0)}{j_{12}(0)+j_{11}(0)}, j=\frac{j_{12}(i, i)}{j_{12}(0)+j_{11}(0)}, \\
& \lambda_{i 1}=\frac{1}{4} j+\eta, \lambda_{i 2}=-\frac{1}{4} j+h_{i}-a \xi, \lambda_{i 3}=-\frac{1}{4} j-h_{i}+a \xi, \lambda_{i 4}=\frac{1}{4} j-\eta .
\end{aligned}
$$

Here all parameters pertaining to energy

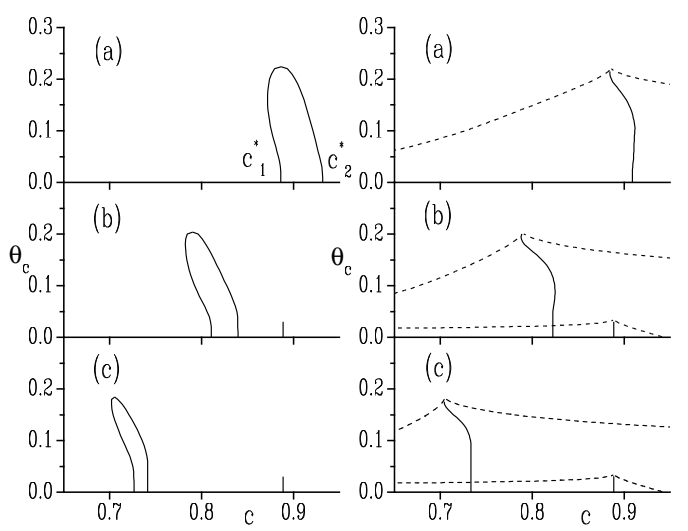

Figure 1. The transition temperature $\Theta_{c}$ as a function of the concentration $c$ (solid lines) at various values of parameters $j$ (on the left panel $j=$ -0.03 ; on the right panel $j=-0.05$ ) and $h$ ( a) $h=0.04$; b) $h=0.08$; c) $h=0.12)$. Values of other parameters are $a=-0.9, h_{\mathrm{vac}}=-0.4$. Dashed lines indicate boundaries of stability regions of metastable phases.

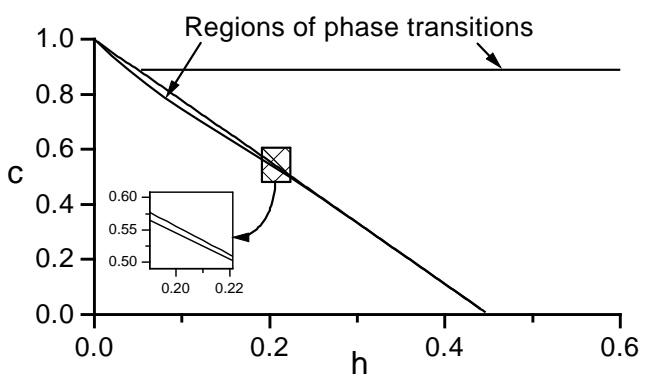

Figure 2. Regions where a first order phase transition between nonpolar phases take place at the change of temperature (most of subregions are of a line width in the picture scale). Values of other parameters are $j=$ $-0.05, a=-0.9, h_{\mathrm{vac}}=-0.4$. are expressed in dimensionless quantities by normalization on $j_{12}(0)+j_{11}(0)$. As a rule the set of simultaneous equations (2.4) has several solutions. It is necessary to take those of them which correspond to the minimum value of the free energy $F$ of the system per site

$$
F=\eta^{2}-a \xi^{2}-\frac{1}{\beta} \sum_{n} p_{n} \ln \sum_{k=1}^{4} \exp \left(\beta \lambda_{n k}\right) .
$$

The ordinary Mitsui model has two phases: a polar phase with noncompensated average pseudospin projections from different sublattices and a nonpolar phase with mutually compensated ones (see [6-8] and references therein). A new feature of the model with random field $h_{i}$ considered here is a possibility of appearing and coexisting of nonequivalent nonpolar phases (average projections of pseudospins in sublattices are compensated in different ways: e.g. $|\uparrow \downarrow\rangle$ or $|\downarrow \uparrow\rangle$ which corresponds to the opposite signs of the average $\xi$ ).

The polar phase differs from the nonpolar qualitatively because they correspond to a different symmetry of the system. They are separated by the first or the second order phase transition line on the phase diagram. Nonpolar phases are of identical symmetry and differ only quantitatively. Thus, only the first order phase transition ending at the critical point, where this difference vanishes, is possible between them and not the average $\xi$ but its deviation $\Delta \xi=\xi-\xi_{\mathrm{c}}$ from the value at the critical point has the meaning of a Landau order parameter. 

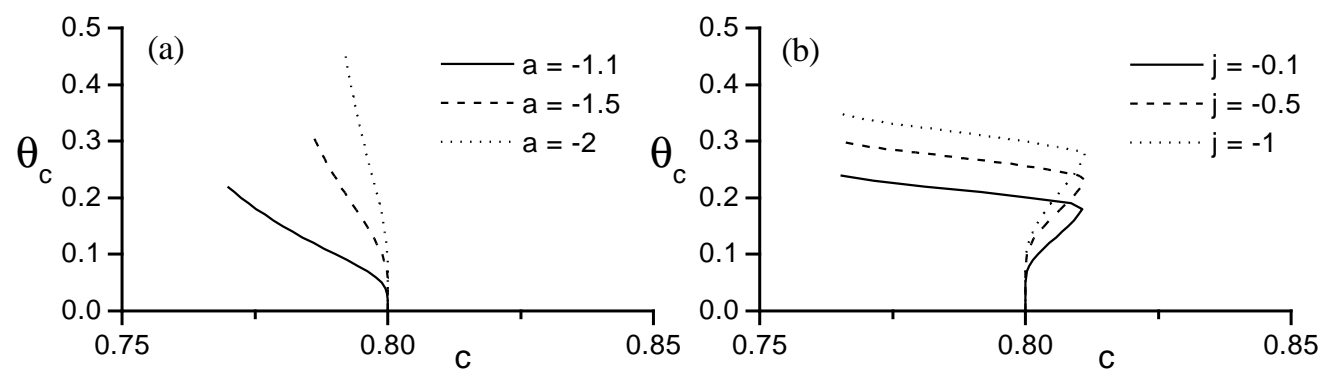

Figure 3. The transition temperature $\Theta_{c}$ as a function of the concentration $c$ at various values of parameters $a$ at $j=0$ (a) and $j$ at $a=-1.1$ (b). Values of other parameters are $h=0.1, h_{\mathrm{vac}}=-0.4$.

Depending on values of the system parameters the phase diagram can undergo qualitative changes. The region of existence of the polar phase narrows not only when $j$ becomes negative but also at $a \rightarrow-1$ and finally as it has been mentioned above a line of a first order phase transition between nonpolar phases appears instead (figure 1). Depending on the values of fields $h$ and $h_{\text {vac }}$ either one (figure 1a) or two (figure 1b,c) regions of polar phases (or lines of the first order phase transition) can exist. A case, when at temperature $\Theta=0$ two nonpolar phases exist and the parameter $\xi$ is equal to $-\frac{1}{2}\left(c<c_{1}^{*}\right)$ or $\frac{1}{2}\left(c>c_{2}^{*}\right)$, is shown on the figure 1a. The increase of the field $h$ value leads to the appearance of an intermediate nonpolar phase with $\xi=c-\frac{1}{2}$ between them. Analysis of the phase diagram at $\Theta=0$ and numerical calculation indicate that the increase of $h$ at the fixed value of $h_{\mathrm{vac}}$ extends the distance between phase transition regions (figure 1b,c). In the vicinity of the first order phase transition line each of the phases can exist as a metastable phase. Boundaries of their stability regions can be obtained by locating the extrema of parameters $\eta$ and $\xi$ dependencies on the concentration $c$ (figure 1). These boundaries also indicate the regions of possible hysteresis phenomena in real systems.

As one can see in figure 1 , for the fixed field $h$ there is a certain region of concentration values, where the system has a first order phase transition at the change of temperature. For a fixed concentration there is an analogical region of the field $h$ values. A joint diagram (figure 2) shows that the regions of $c$ and $h$ values, where phase transitions take place at change of temperature, are narrow.

A subsequent increase of an absolute

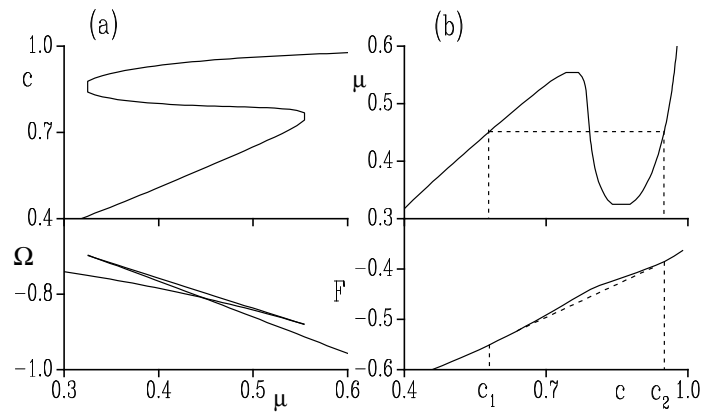

Figure 4. Thermodynamical functions of the system with equilibrium vacancies in the cases $\mu=$ const (a) and $c=$ const (b). Values of parameters are $j=-0.05$, $a=-0.9, h=0.08, h_{\mathrm{vac}}=-0.4, \Theta=$ 0.21 . value of the parameter $a$ strengthens the acting on pseudospins mean field. As a result a concentration region, where the phase transition exists, narrows which is illustrated in figure $3 \mathrm{a}$ at $j=0$ and $a<-1\left(j_{12}<0\right)$. The increase of the parameter $j$ 
absolute value has got a different effect on the shape of the phase diagram (figure 3b); in this case the reentrant phenomena take place which is not surprising for the Mitsui model.

\section{Equilibrium vacancy distribution}

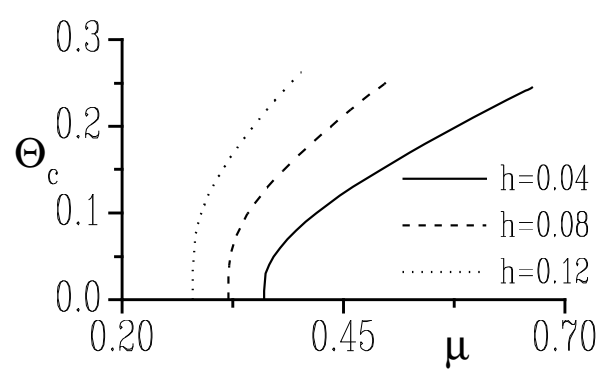

Figure 5. The transition temperature $\Theta_{\mathrm{c}}$ as a function of the chemical potential $\mu$. Values of other parameters are $j=$ $-0.05, a=-0.9, h_{\mathrm{vac}}=-0.4$.

In this case one should explicitly take into account the third sublattice. The longitudinal internal field $h_{i}$ depends here on the occupation number $n_{i}$ of the particle on the site $i$ :

$$
h_{i}=h n_{i}+h_{\mathrm{vac}}\left(1-n_{i}\right) .
$$

Going to the grand canonical ensemble we add to the Hamiltonian (2.1) a term

$$
H^{\prime}=H-\mu \sum_{i} n_{i}
$$

where $\mu$ is the chemical potential of particles.

The basis of states in the cell $\left|S_{1 i}^{z} S_{2 i}^{z} n_{i}\right\rangle$ here additionally includes a particle occupation number. Hence it expands to eight states:

$$
\begin{aligned}
& |1\rangle=|++1\rangle, \quad|2\rangle=|+-1\rangle, \quad|3\rangle=|-+1\rangle, \quad|4\rangle=|--1\rangle, \\
& |\tilde{1}\rangle=|++0\rangle, \quad|\tilde{2}\rangle=|+-0\rangle, \quad|\tilde{3}\rangle=|-+0\rangle, \quad|\tilde{4}\rangle=|--0\rangle .
\end{aligned}
$$

After calculations similar to those made in section 2 the set of equations for parameters $\eta$ and $\xi$ looks like

$$
\left\{\begin{array}{l}
\eta=\frac{1}{2}\left[\exp \left(\beta \lambda_{1}\right)-\exp \left(\beta \lambda_{4}\right)+\exp \left(\beta \lambda_{\tilde{1}}\right)-\exp \left(\beta \lambda_{\tilde{4}}\right)\right]\left[\sum_{k} \exp \left(\beta \lambda_{k}\right)\right]^{-1} \\
\xi=\frac{1}{2}\left[\exp \left(\beta \lambda_{2}\right)-\exp \left(\beta \lambda_{3}\right)+\exp \left(\beta \lambda_{\tilde{2}}\right)-\exp \left(\beta \lambda_{\tilde{3}}\right)\right]\left[\sum_{k} \exp \left(\beta \lambda_{k}\right)\right]^{-1} .
\end{array}\right.
$$

where

$$
\begin{aligned}
& \lambda_{1}=\frac{1}{4} j+\eta+\mu, \lambda_{2}=-\frac{1}{4} j+h_{1}-a \xi+\mu, \lambda_{3}=-\frac{1}{4} j-h_{1}+a \xi+\mu, \lambda_{4}=\frac{1}{4} j-\eta+\mu, \\
& \lambda_{\tilde{1}}=\frac{1}{4} j+\eta, \quad \lambda_{\tilde{2}}=-\frac{1}{4} j+h_{2}-a \xi, \quad \lambda_{\tilde{3}}=-\frac{1}{4} j-h_{2}+a \xi, \quad \lambda_{\tilde{4}}=\frac{1}{4} j-\eta .
\end{aligned}
$$

Average number of particles per site is expressed as

$$
c=\left[\exp \left(\beta \lambda_{1}\right)+\exp \left(\beta \lambda_{2}\right)+\exp \left(\beta \lambda_{3}\right)+\exp \left(\beta \lambda_{4}\right)\right]\left[\sum_{k} \exp \left(\beta \lambda_{k}\right)\right]^{-1} .
$$


Hereinafter the two main cases will be considered: $\mu=$ const, $c$ is calculated from expression (3.5) and $c=$ const, relation (3.5) becomes an additional equation in the set (3.4). Solutions of the set (3.4) with the lowest value of grand thermodynamical potential $\Omega$ per site

$$
\Omega=\eta^{2}-a \xi^{2}-\frac{1}{\beta} \ln \sum_{k} \exp \left(\beta \lambda_{k}\right)
$$

corresponds to the ground state of the system in the case of $\mu=$ const while for $c=$ const the set (3.4) after substitution of $\mu$ from equation (3.5) becomes identical to the set (2.4) and the criterium of the minimal free energy $F=\Omega+\mu n$ is applied.

The basic features of thermodynamics in those cases are illustrated in figure 4 . For $\mu=$ const the system has a first order phase transition at the change of $\mu$ or $h$ (figure 5; the dependence of $\Theta_{\mathrm{c}}$ on $h$ is very similar) or at the change of temperature at certain values of these parameters. For $c=$ const the possibility of the phase transitions at the change of $c$ also exists but they are located inside the phase separation region (figure 6) and hence do not take place (the limits of this region $\left[c_{1}, c_{2}\right]$ can be obtained by applying the Maxwell rule to the dependence $\mu(c)$ or by investigating the values of $c$ at which the free energy $F(c)$ for a phase separated system is lower than for a homogeneous one). Similar behaviour has been reported for the one-sublattice pseudospin-electron model [11].

\section{Conclusions}

Unlike the ordinary Mitsui model the system with the bimodal random field can have various possible phases which correspond to different arrangements of pseudospins: nonequivalent nonpolar phases with mutually compensated pseudospins of different sublattices and polar phase with noncompensated pseudospins.

In the case of nonequilibrium vacancy distribution for certain regions of vacancy concentrations the system has got one or two first order phase transitions between 
nonpolar phases. This first order type of phase transitions leads to a bistable behaviour in real systems. In the case of equilibrium vacancy distribution, $c=$ const the system becomes phase separated in a certain region of parameters.

This model is of practical interest due to the description of the apex oxygen chain oxygen subsystem in nonstoichiometric $\mathrm{YBa}_{2} \mathrm{Cu}_{3} \mathrm{O}_{7-x}$ high- $\mathrm{T}_{\mathrm{c}}$ superconductor.

This work was supported in part by the International Soros Science Education Program through the grant No. SPU 062062 and by the Foundation for Fundamental Investigations of Ukrainian Ministry in Affairs of Science and Technology, project No. 2.4/171.

\section{References}

1. Gasparov L.V., Kulakovskij V.D., Timofeev V.B., Sherman E.Ya. Anharmonicity of bridge oxygen vibration in $\mathrm{YBa}_{2} \mathrm{Cu}_{3} \mathrm{O}_{7-x}$.// JETP, 1991, vol. 100, No. 5(11), p. 16811689 (in Russian).

2. Mustre de Leon J., Conradson S.D., Batistić I., Bishop A.R. Correlation between axial-oxygen anharmonicity and $\mathrm{T}_{c}$ in $\mathrm{YBa}_{2} \mathrm{Cu}_{3} \mathrm{O}_{7}$ and related compounds. // Phys. Rev. B, 1991, vol. 44, No. 5, p. 2422-2425.

3. Palles D., Poulakis N, Liarokapis E., Conder K., Kaldis E., Müller K.A. Raman study of the oxygen anharmonicity in $\mathrm{YBa}_{2} \mathrm{Cu}_{3} \mathrm{O}_{x}(6.4<\mathrm{x}<7.0)$ superconductors. // Phys. Rev. B, 1996, vol. 54, No. 9, p. 6721-6727.

4. Galbaatar T., Plakida N.M. Possible origin of ferroelectricity in $\mathrm{YBa}_{2} \mathrm{Cu}_{3} \mathrm{O}_{7}$ compound. - In Proc. Intern. Conf. M²S-HTSC IV, Grenoble, July 5-9, 1994.

5. Saiko A.P., Gusakov V.E. On the reason of appearance of two superconducting phases in yttrium-barium cuprates. // JETP, 1995, vol. 108, No. 2(8), p. 757-764 (in Russian).

6. Vaks V.G. Introduction in the Microscopical Theory of Ferroelectrics. Moscow, Nauka, 1973 (in Russian).

7. Levitskii R.R., Zachek I.R., Varanitskii V.I. Relaxational dynamics of order-disorder ferroelectric compounds with two minima asymmetrical one-particle potential. // Ukr. Phys. J., 1980, vol. 25, No. 11, p. 1766-1774 (in Russian).

8. Sokolovskii R.O. Thermodynamics, correlation functions and relaxational dynamics of disordered ferroelectric crystals with asymmetric one-particle double-well potential. Preprint of the Institute for Condensed Matter Physics, ICMP-97-10U, Lviv, 1997, 24 p. (in Ukrainian).

9. Stasyuk I.V., Velychko O.V. Influence of oxygen nonstoichiometry on localization of apex oxygens in $\mathrm{YBa}_{2} \mathrm{Cu}_{3} \mathrm{O}_{7-x}$-type crystals. Preprint of the Institute for Condensed Matter Physics, ICMP-98-25E, Lviv, 1998, 17 p.

10. Prelovšek P., Pirc R. CPA approach to the random Ising model in a transverse field. // J. Phys. C, 1977, vol. 10, p. 861-873.

11. Stasyuk I.V., Havrylyuk Yu. Phase transitions in pseudospin-electron model with direct interaction between pseudospins. Preprint of the Institute for Condensed Matter Physics, ICMP-98-18E, Lviv, 1998, 20 p. 


\section{Фазові переходи нового типу в моделі Міцуї в бімодальному випадковому полі}

\section{І.В.Стасюк, О.В.Величко}

Інститут фізики конденсованих систем НАН України, 79011 Львів, вул. Свєнціцького, 1

Отримано 25 грудня 1998 р.

Термодинамічні функції моделі Міцуї в бімодальному випадковому полі отримано в наближенні середнього поля для далекосяжних взаємодій при точному врахуванні взаємодії псевдоспінів у межах комірки. Вивчаються умови появи фазового переходу першого роду між нееквівалентними неполярними фазами та явища фазового розшарування.

Ключові слова: модель Міцуї, випадкове поле, вакансії, фазові переходи, ВТНП

PACS: $64.60 . \mathrm{Cn}, 74.62 . \mathrm{Dh}, 74.72 . \mathrm{Bk}$ 